Aim of the study: The aim was to examine the effects of neoadjuvant chemoradiotherapy on VEGF expression in patients with locally advanced rectal cancer.

Materials and methods: A total of 53 patients with locally advanced rectal cancer were retrospectively studied. Neoadjuvant treatment comprised external beam radiation (50.4 Gy/28 fractions) with continuous infusion of 5 -fluorouracil. Four to 6 weeks after the chemoradiotherapy, the patients underwent surgical resection. Immunohistochemistry was performed to assess VEGF expression in the pretreatment biopsies and in resected specimens.

Results: Resection with microscopic residual tumour (R1) was performed in two patients while in the remain ing 51 patients radical resection with microscopically negative margins (RO) was possible. Downstaging after preoperative chemoradiotherapy was observed in 34 patients (64\%). After chemoradiotherapy 24 patients (45\%) had decreased VEGF expression, in 20 patients (38\%) there was no change, and in two patients it was not possible to assess the dynamics of VEGF expression due to pathologic complete response after chemoradiotherapy. The five-year overall survival (OS) rate was $56 \%$ (95\% Cl: 43-70\%). Although the median OS was 2.5 times shorter in patients who experienced decreased VEGF expression during therapy, this difference did not reach statistical significance. VEGF expression was not significant in Cox regression analysis or log-rank test. VEGF expression decreased after neoadjuvant chemoradiotherapy in most patients with rectal adenocarcinoma examined. This decrease was associated with a trend of inferior prognosis. Conclusions: VEGF expression decreased after neoadjuvant chemoradiotherapy in most patients examined. This decrease was associated with a trend of inferior prognosis.

Key words: rectal adenocarcinoma, neoadjuvant treatment, radiotherapy, chemotherapy, vascular endothelial growth factor.

Contemp Oncol (Pozn) 2017; 21 (1): 48-53 DOI: https://doi.org/10.5114/wo.2017.66656

\section{The changes of tumour vascular endothelial growth factor expression after neoadjuvant chemoradiation in patients with rectal adenocarcinoma}

David Buka ${ }^{1}$, Josef Dvorak ${ }^{2}$, Veronika Sitorova ${ }^{3}$, Igor Sirak ${ }^{1}$, Rene Voboril', Bohuslav Melichar ${ }^{5}$, Igor Richter ${ }^{6}$

\begin{abstract}
'Department of Oncology and Radiotherapy, Charles University, Medical School and Teaching Hospital, Hradec Kralove, Czech Republic

${ }^{2}$ Department of Oncology, First Faculty of Medicine, Charles University and Thomayer Hospital, Prague, Czech Republic

${ }^{3}$ Department of Pathology, Charles University, Medical School and Teaching Hospital, Hradec Kralove, Czech Republic

${ }^{4}$ Department of Surgery, First Faculty of Medicine, Charles University in Prague and $\mathrm{Na}$ Bulovce Hospital, Prague, Czech Republic

DDepartment of Oncology, Palacky University Medical School and Teaching Hospital, Olomouc, Czech Republic

${ }^{6}$ Department of Oncology, Regional Hospital Liberec, Liberec, Czech Republic
\end{abstract}

\section{Introduction}

Total mesorectal excision preceded by a neoadjuvant chemoradiotherapy is the current standard of care for patients with locally advanced rectal cancer [1]. Neoadjuvant chemoradiotherapy using 5-fluorouracil significantly increases the rate of pathologic complete response, lowers the local recurrence rate, but does not improve disease-free survival (DFS) or overall survival (OS) in comparison to external beam radiation alone [2]. Improvement in the long-term outcomes is expected from the incorporation of additional active agents [3].

Vascular endothelial growth factor (VEGF) plays an important role in the development and maintenance of blood vessels as well as in the regulation of vascular endothelial cell permeability [4]. According to immunohistochemical studies VEGF is not expressed in normal colorectal mucosa, but is highly expressed in adenocarcinomas [5-7]. In the sequence of transformation of adenoma to adenocarcinoma VEGF expression represents an early event $[6,7]$. Neovascularisation, supported by increased expression of VEGF, is essential for tumour nutrition and haematogenous spread [8]. In colorectal adenocarcinoma high VEGF expression has been shown to correlate with poor prognosis and higher incidence of liver metastases [7].

In gastrointestinal oncology the inhibition of VEGF is being successfully used in the treatment of metastatic colorectal adenocarcinoma with bevacizumab and aflibercept and in the treatment of advanced gastric or gastro-oesophageal junction adenocarcinoma using ramucirumab. The anti-angiogenic effect of bevacizumab may increase radiosensitivity. Bevacizumab has been incorporated into phase I-II studies of preoperative chemoradiotherapy for rectal cancer, but the toxicity pattern and surgical complications observed in some of these studies were discouraging [3, 9-11]. In addition, there is no predictive biomarker for the therapeutic response to the VEGF inhibitors [12].

The aim of this retrospective study was to evaluate the effect of neoadjuvant chemoradiotherapy on VEGF expression of rectal adenocarcinoma, 
by comparison of VEGF expression in endoscopic biopsies before the initiation of therapy and resection specimens after the therapy. Another aim is to evaluate whether changes in VEGF expression after neoadjuvant treatment influence the survival or treatment response of patients undergoing neoadjuvant chemoradiotherapy for rectal adenocarcinoma.

\section{Material and methods}

\section{Patient characteristics}

Fifty-three patients, 38 males and 15 females, median age 63 (range 41-75) years, with locally advanced rectal adenocarcinoma were retrospectively studied. Sixteen patients had clinical stage II and 37 patients had clinical stage

Table 1. Patient characteristics with VEGF expression score before and after preoperative radiochemotherapy

\begin{tabular}{|c|c|c|c|c|c|c|}
\hline $\begin{array}{l}\text { Patient } \\
\text { number }\end{array}$ & Age & Gender & Clinical stage & $\begin{array}{l}\text { VEGF expression score } \\
\text { in pretreatment biopsies }\end{array}$ & Pathological stage & $\begin{array}{l}\text { VEGF expression score } \\
\text { in resected specimens }\end{array}$ \\
\hline 1 & 64 & $\mathrm{~F}$ & сT3CNOMO & 0 & урТЗурNOMO & 1 \\
\hline 2 & 57 & $M$ & cT3cN1MO & 3 & урT3урN1M0 & 1 \\
\hline 3 & 71 & $M$ & cT3cN1M0 & 3 & урT3урNOMO & 3 \\
\hline 4 & 72 & $\mathrm{~F}$ & cT3cN1M0 & 2 & урT3урNOMO & 1 \\
\hline 5 & 60 & $M$ & cT3cN1M0 & 2 & урТ1урNOMO & 2 \\
\hline 6 & 60 & $\mathrm{~F}$ & cT3cN1M0 & 2 & урT2ypNOMO & 1 \\
\hline 7 & 53 & $\mathrm{~F}$ & CT3CN1MO & 3 & урТЗурNOMO & 2 \\
\hline 8 & 66 & $M$ & cT3cNOMO & 2 & урТЗурNOMO & 2 \\
\hline 9 & 63 & $M$ & сT3cN1M0 & 3 & урТЗурNOMO & 2 \\
\hline 10 & 59 & $M$ & сT3cN1M0 & 3 & урT3ypN1M0 & 1 \\
\hline 11 & 70 & $M$ & сT3cNOMO & 3 & урT3урNOMO & 1 \\
\hline 12 & 62 & $\mathrm{~F}$ & cT3cNOMO & 2 & урT2ypNOMO & 2 \\
\hline 13 & 75 & $M$ & сT3CNOMO & 3 & урТЗурNOMO & 1 \\
\hline 14 & 74 & $M$ & cT4CN1MO & 2 & урТзурNOMO & 1 \\
\hline 15 & 58 & $M$ & CT3CNOMO & 2 & урT3урN1M0 & 2 \\
\hline 16 & 67 & $\mathrm{~F}$ & сT3cNOMO & 3 & урТЗурNOMO & 3 \\
\hline 17 & 67 & $M$ & cT3CN1M0 & 1 & урT3ypN1M0 & 1 \\
\hline 18 & 53 & $M$ & cT4cN1M0 & 1 & ypT3ypNOM1 (liver) & 1 \\
\hline 19 & 58 & $M$ & CT3CNOMO & 3 & урT2ypNOMO & 1 \\
\hline 20 & 71 & $M$ & cT4CN1M0 & 2 & урT2ypNOMO & 1 \\
\hline 21 & 75 & $M$ & cT3cN1MO & 3 & урТЗурNOMO & 3 \\
\hline 22 & 67 & $M$ & cT3cN1MO & 3 & ypT2ypNOMO & 2 \\
\hline 23 & 70 & $M$ & cT3CN1M0 & 3 & урT3ypN1M0 & 1 \\
\hline 24 & 60 & $M$ & сT3cNOMO & 2 & урТЗурNOMO & 3 \\
\hline 25 & 64 & $M$ & cT4CN1MO & 1 & урТЗурNOMO & 2 \\
\hline 26 & 67 & $\mathrm{~F}$ & cT4CN1M0 & 3 & урT3урN1M0 & 2 \\
\hline 27 & 60 & $M$ & cT4cN1M0 & 3 & урT2ypNOMO & 2 \\
\hline 28 & 67 & $M$ & cT3cN1M0 & 3 & урT3урNOMO & 2 \\
\hline 29 & 50 & $M$ & сT3cNOMO & 3 & урT3урNOMO & 1 \\
\hline 30 & 64 & $\mathrm{~F}$ & cT4cN1MO & 3 & ypT4ypNOM1 (liver) & 1 \\
\hline 31 & 72 & $M$ & cT3cN1M0 & 2 & ypT3ypNOM1 (liver) & 1 \\
\hline 32 & 64 & $M$ & cT3cNOMO & 2 & ypT2ypN1M0 & 2 \\
\hline 33 & 61 & $M$ & cT3cN1M0 & 3 & урT2урNOMO & 1 \\
\hline 34 & 57 & $M$ & cT3cNOMO & 3 & урT2ypNOMO & 3 \\
\hline 35 & 74 & $M$ & cT3cN1M0 & 2 & ypTOypNOMO pCR & - \\
\hline 36 & 75 & $\mathrm{~F}$ & cT3cN1M0 & 2 & ypTOypNOMO pCR & - \\
\hline 37 & 70 & $M$ & cT3cN1M0 & 2 & урT3урN1M0 & 2 \\
\hline 38 & 50 & $M$ & сT3cN1MO & 3 & урT2ypNOMO & 3 \\
\hline 39 & 72 & $\mathrm{~F}$ & cT3cN1M0 & 3 & урТ3урN1M0 & 2 \\
\hline
\end{tabular}


Table 1. Cont.

\begin{tabular}{|c|c|c|c|c|c|c|}
\hline $\begin{array}{l}\text { Patient } \\
\text { number }\end{array}$ & Age & Gender & Clinical stage & $\begin{array}{l}\text { VEGF expression score } \\
\text { in pretreatment biopsies }\end{array}$ & Pathological stage & $\begin{array}{l}\text { VEGF expression score } \\
\text { in resected specimens }\end{array}$ \\
\hline 40 & 59 & M & cT3cN1MO & 2 & урТ3урN1M0 & 3 \\
\hline 41 & 64 & $\mathrm{~F}$ & $\mathrm{CT3} \mathrm{CN} 1 \mathrm{MO}$ & 3 & ypT2ypNOMO & 3 \\
\hline 42 & 58 & $F$ & CT3CN1MO & 1 & ypTlypNOMO & 3 \\
\hline 43 & 75 & $F$ & CT3CN1MO & 3 & урT3урNOMO & 3 \\
\hline 44 & 61 & M & cT3cN1M0 & 2 & ypT2ypNOMO & 3 \\
\hline 45 & 69 & M & CT3CNOMO & 1 & $\begin{array}{l}\text { ypTlypNOMO } \\
\text { (micr. res. tu) }\end{array}$ & 0 \\
\hline 46 & 63 & M & cT3cN1MO & 2 & $\begin{array}{r}\text { ypTlypNOMO } \\
\text { (micr. res. tu) }\end{array}$ & 2 \\
\hline 47 & 41 & M & cT3cN1MO & 2 & урT3урNOMO & 2 \\
\hline 48 & 61 & M & CT3CNOMO & 2 & урT3урNOMO & 2 \\
\hline 49 & 63 & M & cT4cN1M0 & 3 & урT1ypN1MO & 2 \\
\hline 50 & 41 & $\mathrm{~F}$ & $\mathrm{cT} 2 \mathrm{cN} 1 \mathrm{MO}$ & 3 & урT2урN1M0 & 3 \\
\hline 51 & 59 & M & CT3CNOMO & 3 & ypT1ypNOMO & 2 \\
\hline 52 & 42 & $\mathrm{~F}$ & CT3CNOMO & 2 & ypT1ypNOMO & 2 \\
\hline 53 & 54 & M & cT3CN1MO & 2 & ypT2ypNOMO & 3 \\
\hline
\end{tabular}

M-male; F-female; cTCNM - pretreatment clinical TNM classification; ypTypNM - postsurgical histopathological TNM classification after neoadjuvant chemoradiotherapy; $p C R$ - pathological complete response; micr. res. tu-microscopic residual tumour

III tumours (Table 1). The anatomical localisation was as follows: upper rectum $(>10 \mathrm{~cm})$ in 6 patients, middle rectum $(>5-10 \mathrm{~cm})$ in 28 patients, and lower rectum $(<5 \mathrm{~cm}$ from the anal verge) in 19 patients. All patients had, in a pretreatment biopsy, histologically verified adenocarcinoma (grade 1 in 3 patients, grade 2 in 32 patients, and grade 3 in 18 patients). The tumour grade in the resection specimen was as follows: three patients had grade 1, 28 patients had grade 2 , and 20 patients had grade 3 . The median pretreatment CEA level was 4.02 (range 0.39-62.39) $\mu \mathrm{g} / \mathrm{l}$.

\section{Treatment}

Neoadjuvant chemoradiotherapy comprised external beam radiation with concomitant continuous infusion of 5 -fluorouracil $200 \mathrm{mg} / \mathrm{m}^{2} /$ day throughout the course of radiation, which was interrupted during the weekends.

Pelvic radiotherapy was delivered for all patients using megavoltage photon beams (6 or $15 \mathrm{MV}$ ) from a linear accelerator (Clinac 600, Clinac 2100, or Varian, Varian Medical Systems, Palo Alto, CA, USA). All fields were irradiated daily, five days per week. In total, a dose of 45 Gy in 25 fractions (single dose $1.8 \mathrm{~Gy}$ ) was administered to the intersection of the fields. The boost volume was treated to a dose of 5.4 Gy in three fractions (single dose $1.8 \mathrm{~Gy}$ ).

Surgery was performed 4-6 weeks after the end of chemoradiotherapy.

The Common Toxicity Criteria for Adverse Events version 4.0 was applied to evaluate toxicity.

\section{Immunohistochemical determination of VEGF}

The immunohistochemical evaluation was scored semi-quantitatively, and the cytoplasmatic intensity of at least $1 \%$ of invasive tumour cells was assessed as follows: $0=$ none, $1+=$ weak staging $($ Fig. $1 \mathrm{~A}), 2+=$ moderate stag- ing (Fig. 1B), or 3+ = strong staining (Fig. 1C, Denmark) was used. Slides were evaluated by an experienced pathologist who was not familiar with the treatment results of the patients. Endobioptic findings before treatment as well as resection specimens after neoadjuvant chemoradiotherapy and surgical treatment were analysed in this patient study.

\section{Statistical analysis methods}

The statistical evaluation was performed using the Number Cruncher Statistical Systems 9 NCSS (Kaysville, UT, USA) program. OS and DFS were assessed using Kaplan-Meier analysis. The effect of VEGF expression on treatment outcomes was assessed by the log-rank test and Cox regression analysis. The predictive impact of the changes in VEGF expression and treatment response was evaluated using the $\chi^{2}$ test. In each case the decision on statistical significance was based at the significance level $\alpha=0.05$.

\section{Results}

All patients completed the planned dose of radiotherapy. Two patients did not complete the planned dose of chemotherapy, in both cases due to haematological toxicity. There was no treatment-related mortality, and preoperative chemoradiotherapy was reasonably well tolerated in the majority of cases. Grade 3 diarrhoea occurred in three patients and grade 3 leukopenia in two patients. The median nadir of haemoglobin concentration was 124 (range 92-165) g/l, median nadir of leukocyte count was 4.2 (range 1.6-14.5) $10^{9} /$, and median nadir platelet count was 198 (range 93-263) 10\%/l. Radical resection with microscopically negative margins (RO) was performed in 51 patients (sphincter-preserving resection in 22 patients and abdominoperineal resection in 29 patients) and resection with microscopic residual tumour (R1) in two patients (one 

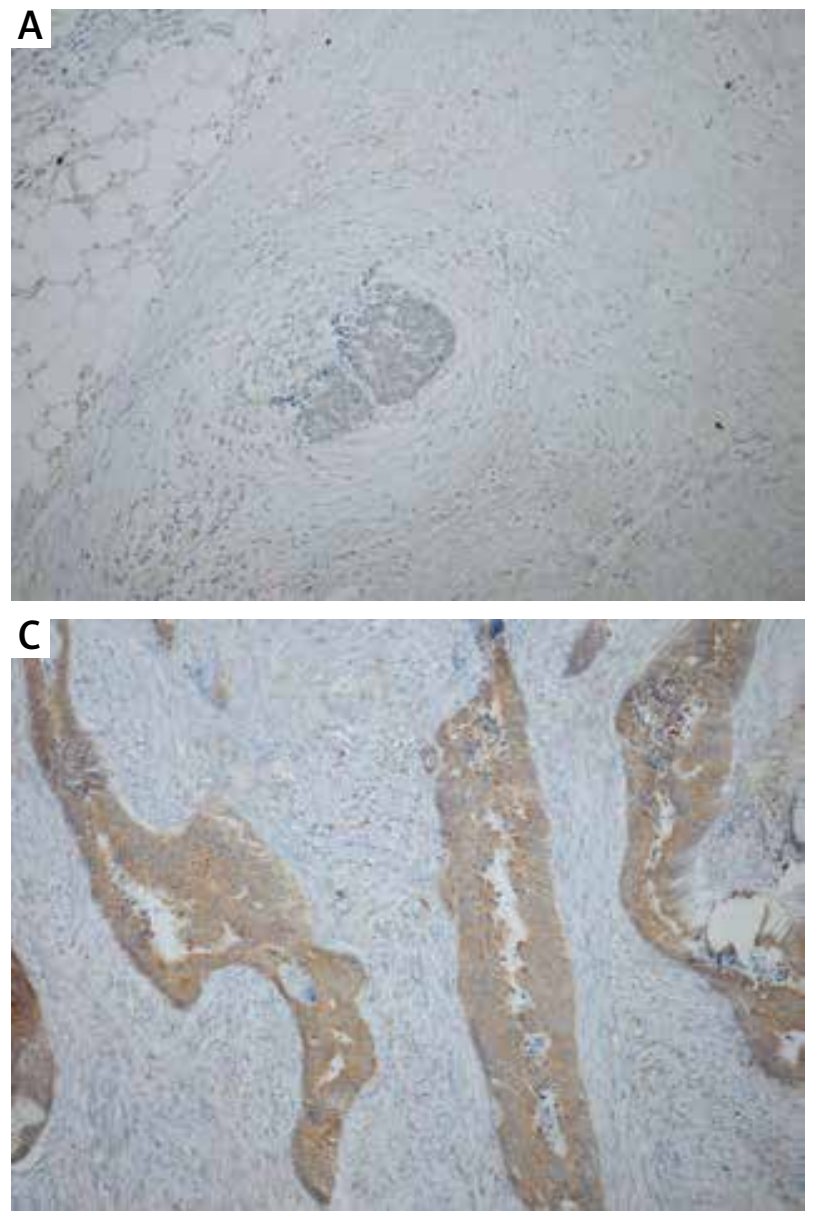

patient after sphincter-preserving resection, and one patient after abdominoperineal resection). Based on TNM definitions, the pathologic stage was as follows: pCR two patients, microscopic residual tumour two patients, stage | 16 patients, stage I| 18 patients, stage I|| 12 patients, and stage IV three patients. All three patients with stage IV had liver metastases that were detected during surgery. Downstaging after preoperative chemoradiotherapy was observed in 34 patients (64\%). In the resection specimen after chemoradiotherapy, VEGF expression decreased in 24 patients (45\%), increased in seven patients (13\%), did not change in 20 patients (38\%), and in two patients the change was not evaluable because of pathologic complete response (Tables 1 and 2). Interestingly, VEGF expression levels increased only in patients with Score 1 and decreased only in patients with Score 3.

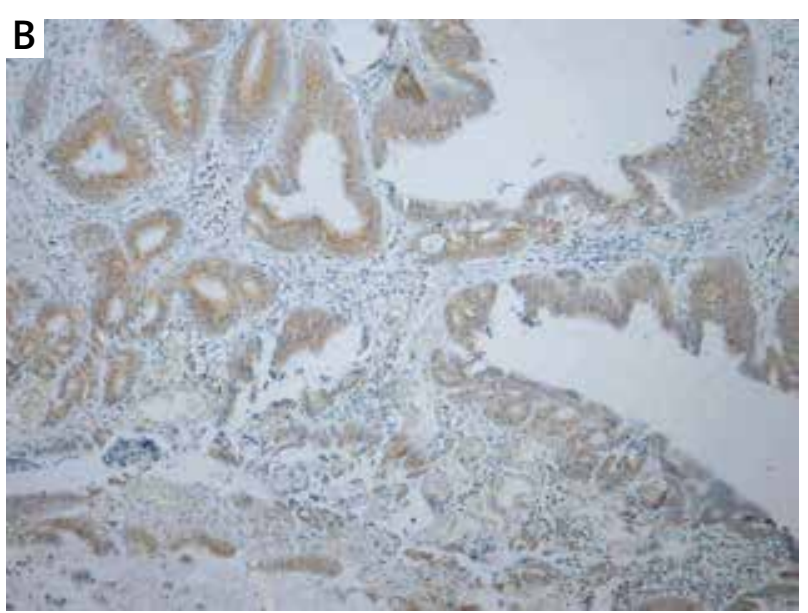

Fig. 1. A) VEGF staining score 1+; B) VEGF staining score 2+; C) VEGF staining score $3+$

The median follow-up was 109 months (9.1 years). During the follow-up 24 patients had recurrence and 30 patients died. At the time of this analysis, 22 patients were alive with no recurrence and one patient was alive with recurrence. Five-year OS rate was 56\% (95\% Cl: 43$70 \%$ ) with median OS of 8.6 years (Fig. 2). Median OS was 2.5 times shorter in patients who experienced decreased VEGF expression during therapy, but this difference did not reach statistical significance (Fig. 3). VEGF expression was not significant in Cox regression analysis or log-rank test. The coefficient of determination (R2) in Cox regression analysis was 0.0267. R2 in analysis of deviance was not significant $(p=0.502)$. Similarly, decrease in VEGF expression after neoadjuvant treatment was not predictive for treatment response and tumour downstaging $(p=0.61)$.

Table 2. Vascular endothelial growth factor (VEGF) expression score in pretreatment biopsies and resected specimens

$\begin{array}{lcc}\text { VEGF expression } & \text { Pretreatment biopsies (patients) } & \text { Resected specimens (patients) } \\ \text { Score } 0 & 1(2 \%) & 1(2 \%) \\ \text { Score } 1+ & 5(9 \%) & 17(32 \%) \\ \text { Score } 2+ & 21(40 \%) & 20(38 \%) \\ \text { Score } 3+ & 26(49 \%) & 13(24 \%) \\ \text { PCR after chemoradiation } & & 2(4 \%)\end{array}$

$p C R$ - pathological complete response 


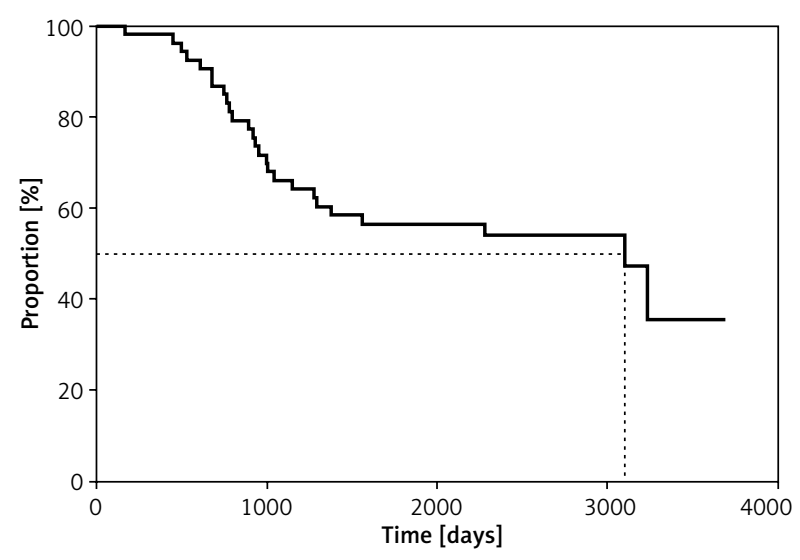

Fig. 2. Kaplan-Meier overall survival curve in days (solid line) with median overall survival (dotted line)

\section{Discussion}

In the present study, we observed differential response of VEGF expression to neoadjuvant chemoradiation, with a decreased expression observed in most patients. A decrease of VEGF expression was associated with a trend of worse survival. It cannot be excluded that in a larger cohort of patients the predictive or prognostic role of VEGF expression would be more evident and could reach statistical significance. It is very interesting that the prognosis is lower when VEGF decreases. This should be taken into account, in view of the damages due to the destruction of angiogenesis by antiangiogenic agents. Because the results of this study indicate an association between a decrease of VEGF during the preoperative chemoradiotherapy, it may be hypothesised that the inhibition of angiogenesis using bevacizumab during preoperative chemoradiotherapy for rectal cancer may be contra productive.

The first concept of antiangiogenic therapy aimed to destroy tumour vessels, while it turned out that, paradoxically, antiangiogenic drugs normalised vasculature and as a result offered an improvement in chemotherapeutic delivery $[13,14]$. The results of the present study support the positive importance of the normalisation of tumour vasculature rather than destruction.

A major limitation of the present pilot retrospective study is a relatively small number of patients. The size of this cohort was limited by the intention to analyse a more homogeneous cohort of patients treated at one centre using the same chemoradiotherapy regimen and same surgical management and pathological processing of samples. The subgroup of patients with no decrease of VEGF expression was heterogeneous and included patients with pathological complete response, which could have contributed to the favourable outcome. Thus, the hypothesis that the decrease of VEGF expression after chemoradiation is associated with inferior prognosis should be investigated in a large prospective cohort of patients.

The observation of decreased VEGF expression after chemoradiation is in contrast with an earlier study that examined VEGF expression before and after preopera-

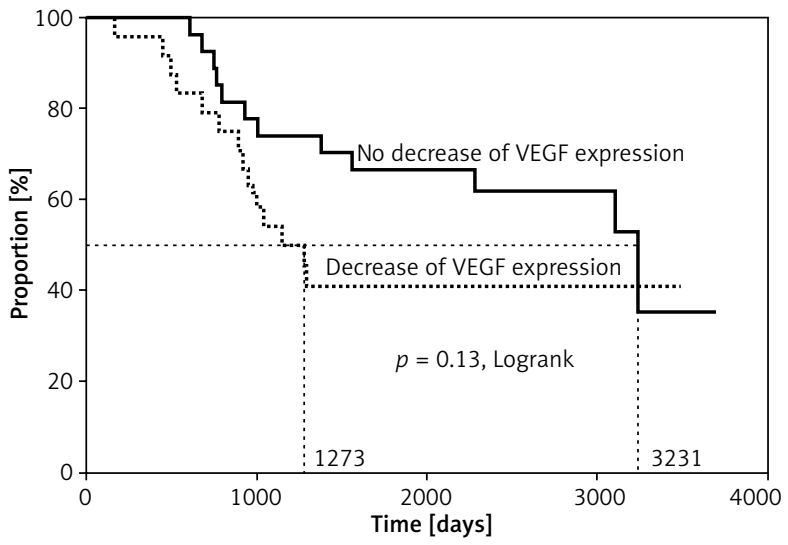

Fig. 3. No decrease of VEGF expression overall survival curve (solid line)

tive radiotherapy was examined in a study investigating 16 locally advanced rectal cancer patients. The tissues biopsied before preoperative radiotherapy and resection specimens were immunohistochemically stained. Four cases were negative for VEGF expression before radiotherapy, but the 12 other cases were positive, with four of these demonstrating strong immunoreactivity. After radiotherapy, all but one case demonstrated VEGF-positive patterns, in which 14 cases showed marked staining. VEGF expression increased after radiotherapy in 12 cases, compared to a single case that showed reduced expression [15]. In the present study we combined radiotherapy with concomitant administration with 5 -fluorouracil and examined higher number of patients.

VEGF expression was examined also in two studies before and after preoperative high-dose rate brachytherapy 26 Gy/4 fractions/4 days for local advanced rectal cancer $[7,16]$. In the present study we used a preoperative chemoradiotherapy schedule, which is more commonly employed in routine clinical practice than preoperative brachytherapy.

In three similar retrospective studies it was observed that the overexpression of epidermal growth factor receptor (EGFR) during neoadjuvant chemoradiotherapy for locally advanced rectal adenocarcinoma, assessed by comparison of EGFR expression in endoscopic biopsies before, and resection specimens after the therapy, is associated with significantly shorter overall survival and disease-free survival [17-19]. However, such an association with outcome could not be demonstrated in the present study for VEGF.

In conclusion, VEGF expression decreased after neoadjuvant chemoradiotherapy in most patients with rectal adenocarcinoma examined in the present study. This decrease was associated with a trend of inferior prognosis that did not reach statistical significance.

This article was supported by research program Progres Q40/06 of Charles University in Prague.

The authors declare no conflict of interest. 


\section{References}

1. Sauer R, Liersch T, Merkel S, et al. Preoperative versus postoperative chemoradiotherapy for rectal cancer: results of the German CAO/ARO/AIO-94 randomised phase III trial after a median follow-up of 11 years. J Clin Oncol 2012; 30: 1923-33.

2. Yaffee P, Osipov A, Tan C, Tuli R, Hendifar A. Review of systemic therapies for locally advanced and metastatic rectal cancer. J Gastrointest Oncol 2015; 6: 185-200.

3. Nussbaum N, Altomare I. The neoadjuvant treatment of rectal cancer: a review. Curr Oncol Rep 2015; 17: 434. doi: 10.1007/s11912 014-0434-9.

4. Breen EC. VEGF in biological control. J Cell Biochem 2007; 102 1358-67.

5. Kang SM, Maeda K, Onoda N, Chung YS, Nakata B, Nishiguchi Y, Sowa M. Combined analysis of p53 and vascular endothelial growth factor expression in colorectal carcinoma for determination of tumor vascularity and liver metastasis. Int J Cancer 1997; 74: 502-07.

6. Wong MP, Cheung N, Yuen ST, Leung SY, Chung LP. Vascular endothelial growth factor is up-regulated in the early pre-malignant stage of colorectal tumour progression. Int J Cancer 1999; 81: 845 50.

7. Zlobec I, Steele R, Compton CC. VEGF as a predictive marker of rectal tumor response to preoperative radiotherapy. Cancer 2005; 104: 2517-21.

8. Theodoropoulos GE, Lazaris AC, Theodoropoulos VE, Papatheodosiou K, Gazouli M, Bramis J, Patsouris E, Panoussopoulos D. Hypoxia, angiogenesis and apoptosis markers in locally advanced rectal cancer. Int J Colorectal Dis 2006; 21: 248-57.

9. Glynne-Jones R, Hadaki M, Harrison M. The status of targeted agents in the setting of neoadjuvant radiation therapy in locally advanced rectal cancers. J Gastrointest Oncol 2013; 4: 264-84.

10. Marquardt F, Rödel F, Capalbo G, Weiss C, Rödel C. Molecular targeted treatment and radiation therapy for rectal cancer. Strahlenther Onkol 2009; 185: 371-8.

11. Torino F, Sarmiento R, Gasparini G. The contribution of targeted therapy to the neoadjuvant chemoradiation of rectal cancer. Crit Rev Oncol Hematol 2013; 87: 283-305.

12. Willett CG, Boucher Y, di Tomaso, et al. Direct evidence that the VEGF-specific antibody bevacizumab has antivascular effects in human rectal cancer. Nat Med 2004; 10: 145-7.

13. Maj E, Papiernik D, Wietrzyk J. Antiangiogenic cancer treatment: The great discovery and greater complexity (Review). Int J Oncol 2016; 49: 1773-84.

14. Lin Z, Zhang Q, Luo W. Angiogenesis inhibitors as therapeutic agents in cancer: Challenges and future directions. Eur J Pharmacol 2016; 793: 76-81.

15. Nozue M, Isaka N, Fukao K. Over-expression of vascular endothelial growth factor after preoperative radiation therapy for rectal cancer. Oncol Rep 2001; 8: 1247-9.

16. Zlobec I, Vuong T, Compton CC, Lugli A, Michel RP, Hayashi S, Jass JR. Combined analysis of VEGF and EGFR predicts complete tumour response in rectal cancer treated with preoperative radiotherapy. Br J Cancer 2008; 98: 450-6.

17. Dvorak J, Sitorova V, Ryska A, et al. The prognostic significance of changes of tumor epidermal growth factor receptor expression after neoadjuvant chemoradiation in patients with rectal adenocarcinoma. Strahlenther Onkol 2012; 188: 833-8.

18. Richter I, Dvorak J, Urbanec M, Bluml A, Cermakova E, Bartos J, Petera J. The prognostic significance of tumor epidermal growth factor receptor (EGFR) expression change after neoadjuvant chemoradiation in patients with rectal adenocarcinoma. Contemp Oncol (Pozn) 2015; 19: 48-53.

19. Richter I, Dvorak J, Bluml A, et al. Influence of preoperative chemoradiotherapy on changes of epidermal growth factor receptor expression in patients treated by preoperative chemoradiotherapy for local advanced rectal carcinoma. Klin Onkol 2014; 27: 361-6.

\section{Address for correspondence}

\section{David Buka}

Department of Oncology and Radiotherapy

Charles University, Medical School and Teaching Hospital Hradec Kralove, Czech Republic

e-mail: davidbuka@seznam.cz

\author{
Submitted: 16.03 .2016
}

Accepted: $\quad 29.01 .2017$ 